\title{
Staphylococcus epidermidis endophthalmitis following intraocular lens implantation
}

\author{
DAVID J. SCHANZLIN, DANIEL B. GOLDBERG, AND STUART I. BROWN \\ From the Department of Ophthalmology, University of Pittsburgh, \\ Pittsburgh School of Medicine and Eye and Ear Hospital, USA
}

SUMMARY A 90-year-old man developed a hypopyon following cataract extraction with intraocular lens implantation. The hypopyon cleared with topical corticosteroid therapy but recurred whenever the corticosteroid therapy was reduced. At surgery for the removal of the intraocular lens an opaque anterior vitreous membrane was excised. Cultures of the anterior vitreous grew Staphylococcus epidermidis. The diagnosis was further confirmed by the histology of the anterior vitreous membrane, which showed Gram-positive cocci in the macrophages and polymorphonuclear leucocytes. This case shows that corticosteroids may completely mask an endophthalmitis from an organism of low virulence such as Staphylococcus epidermidis. Endophthalmitis from an organism of low virulence should be considered in any case of persistent postoperative inflammation.

Before 1973 the significance of culturing Staphylococcus epidermidis from the conjunctiva or aqueous humour in cases of endophthalmitis was disputed. ${ }^{1}$ In 1973 Valenton et al. ${ }^{2}$ reported 2 cases of $S$. epidermidis endophthalmitis where the diagnosis was confirmed by cytology and culture of aqueous removed by anterior chamber paracentesis. Forster and associates ${ }^{3}$ in 1976 reported a large series of cases with postoperative endophthalmitis and showed a high incidence of positive vitreous cultures for $S$. epidermidis. Five of the 6 eyes from this series which eventually attained useful visual acuity were cases of endophthalmitis caused by $S$. epidermidis. More recently Eichenbaum and associates ${ }^{4}$ reported 6 cases of $S$. epidermidis endophthalmitis in eyes following intraocular lens implantation.

We report herein a case of Staphylococcus epidermidis endophthalmitis occurring after intraocular lens implantation which masqueraded as a sterile endophthalmitis because it initially resolved with topical corticosteroid therapy.

\section{Case report}

A 90-year-old man underwent an uncomplicated right intracapsular lens extraction with the implantation of a 4-loop Binkhorst intraocular lens in

Correspondence to Dr Stuart I. Brown, Department of Ophthalmology, Eye and Ear Hospital, 230 Lothrop St, Pittsburgh, Pennsylvania 15213, USA.
June 1978. The immediate postoperative period was uncomplicated. On the fifth postoperative day a mild inflammation was observed in the anterior chamber. This inflammation progressed to a $3 \mathrm{~mm}$ hypopyon by the seventh postoperative day. The patient was treated with a subconjunctival injection of gentamicin $(20 \mathrm{mg})$ and topically applied dexamethasone $0 \cdot 1 \%$ and gentamicin $(3 \mathrm{mg} / \mathrm{ml})$. The hypopyon was reduced within 24 hours and resolved 3 days after this treatment was initiated. The topical antibiotics were reduced while the topical dexamethasone was continued hourly because of the persistent cellular reaction in the anterior chamber. When the topical dexamethasone $0 \cdot 1 \%$ was tapered to every 3 hours the hypopyon returned. The frequency of the topical corticosteroid was again increased to every hour, with complete resolution of the hypopyon within 48 hours. When the topical corticosteroid therapy was tapered to less than every hour, however, the hypopyon returned again, and again with hourly corticosteroid the hypopyon resolved completely within 48 hours.

The patient was seen in consultation in August 1978, 10 weeks postoperatively. At that time he was being treated with hourly topical dexamethasone $0 \cdot 1 \%$. Ocular examination revealed a visual acuity of light perception with good light projection OD and $20 / 80 \mathrm{OS}$. The intraocular pressure was $8 \mathrm{mmHg}$ OD and $21 \mathrm{mmHg}$ OS. There was a $2+$ cellular reaction and heavy flare in the anterior chamber, but no hypopyon was present. A few scattered 


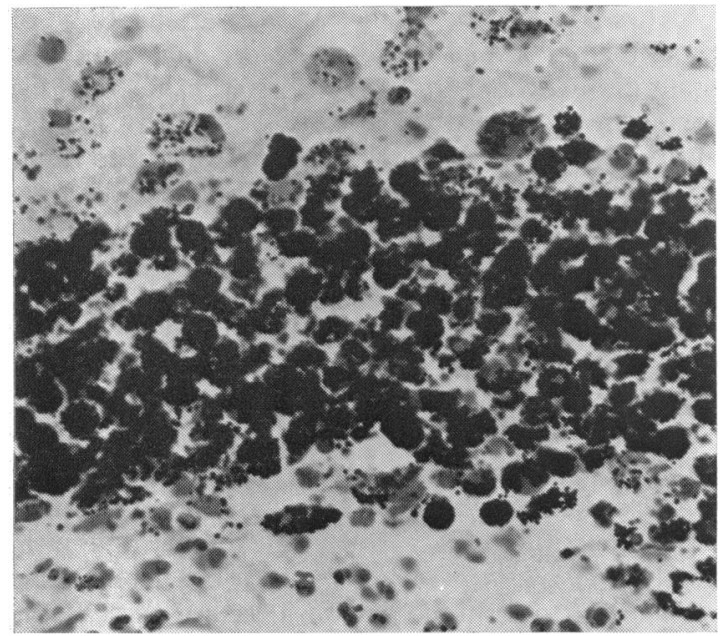

Fig. 1 Gram-stained smear of the anterior vitreous excised from the eye with endophthalmitis caused by Staphylococcus epidermidis. Numerous intracellular cocci are seen within the macrophages and polymorphonuclear leucocytes. $(\times 215)$.

keratic precipitates were on the central corneal endothelium. A 4-loop Binkhorst intraocular lens was in place, and there were posterior synechiae to a partially organised vitreous membrane, which was located behind the intraocular lens. Ultrasonography revealed an apparently normal posterior pole.

The condition was diagnosed as a postoperative uveitis secondary either to the intraocular lens or to a low-grade endophthalmitis. Since the patient had no hypopyon on initial examination, the topical dexamethasone $0.1 \%$ was decreased to every 2 hours and topical gentamicin $(3 \mathrm{mg} / \mathrm{ml})$ was initiated. Three days later a hypopyon was present. On the following day, after an anterior chamber tap, the intraocular lens was removed, revealing a semiopaque anterior vitreous. The anterior vitreous was partially excised and sent for histological and bacteriological analysis.

Trypticase broth cultures of the anterior chamber aspirate were sterile. However, cultures of the anterior vitreous grew Staphylococcus epidermidis (coagulase-negative, mannitol-negative) sensitive to penicillin, cephalothin, ampicillin, erythromycin, and neomycin. In addition, Gram and Giemsa stains of the anterior vitreous membrane revealed numerous macrophages and polymorphonuclear leucocytes with intracellular Gram-positive cocci (Fig. 1).

Postoperatively the patient was treated with oral dicloxocillin $500 \mathrm{mg} 4$ times per day, topical gentamicin $(3 \mathrm{mg} / \mathrm{ml})$ every 2 hours, topical Neo- sporin (polymyxin B, neomycin, and gramicidin) every 2 hours, and topical dexamethasone $0.1 \%$ every 3 hours. The anterior chamber reaction cleared by the end of the first postoperative week, and the vitreous cleared one week later, allowing visualisation of a macular scar with overlying traction bands. Systemic and topical medications were gradually reduced, and the visual acuity returned to $20 / 200$.

\section{Discussion}

Bacterial endophthalmitis usually presents 48 hours or more after cataract extraction, producing pain, decreased light perception and projection, anterior chamber reaction, vitreous reaction, and hypopyon. ${ }^{5}$ It must be differentiated from toxic iritis and sterile iritis, which may also cause a severe anterior chamber reaction in the first postoperative week. Toxic iritis presents in the first 24 hours after surgery and results from the introduction into the eye of disinfectants, soaps, or plastic products which have been sterilised with ethylene oxide. Sterile iritis may be observed at any time in the first postoperative week presenting with pain, anterior chamber reaction, vitreous reaction, and sometimes hypopyon. Fibrin and membranous opacification of the anterior hyaloid face commonly occurs in such cases. Sterile iritis is caused by trauma to the iris and ciliary body during the extraction of the lens. Both toxic iritis and sterile iritis respond rapidly to topical mydriatic and corticosteroid therapy, whereas a bacterial endophthalmitis will not do so. In 1976 Allen and Grove ${ }^{5}$ proposed that a reduction of postoperative inflammation within 24 hours after the initiation of topical corticosteroid and mydriatic treatment serves to differentiate an aseptic iritis from a bacterial endophthalmitis.

Early studies on the incidence of bacterial endophthalmitis following cataract extrastion failed to demonstrate Staphylococcus epidermidis as a causative organism. ${ }^{5}$ Since 1973 , however, there have been several reports of postoperative endophthalmitis caused by $S$. epidermidis, ${ }^{1-4}$ and the pathogenicity of this organism is now accepted. In recent reports ${ }^{4}$ $S$. epidermidis appears to be a significant cause of endophthalmitis following intraocular lens implantation.

Staphylococcus epidermidis is the commonest organism cultured from the conjunctivial sac at the time of cataract extraction. ${ }^{6}$ The increased surgical manipulation associated with intraocular lens implantation may cause an increased intraocular inoculum of it during the operation. This may explain the relatively frequent occurrence of endoph- 
thalmitis due to this organism after lens implantation.

The patient presented here is another case of Staphylococcus epidermidis endophthalmitis following intraocular lens implantation. As with many of Eichenbaum et al.' $\mathrm{s}^{4}$ cases, the onset of the postoperative inflammation in our patient did not begin until late in the first postoperative week. Similarly, our patient was initially misdiagnosed as a sterile iritis.

The prompt resolution of the anterior chamber inflammation with topical corticosteroids was initially suggestive of a sterile inflammatory process. The persistence of the inflammation and the recurrence of the hypopyon whenever the corticosteroids were decreased ruled against a sterile process and suggested an endophthalmitis due to an organism of low virulence. Surgical removal of the intraocular lens revealed an organised anterior vitreous membrane. Cultures of the aqueous humour failed to yield any organism, though this may have been due to the topical antibiotic therapy. Cultures of the anterior vitreous, however, grew Staphylococcus epidermidis. The significance of this culture was further confirmed by cytology of the anterior vitreous membrane, which showed numerous macrophages and polymorphonuclear leucocytes with intracellular Gram-positive cocci. To our knowledge this is the first case report describing the histology of an anterior vitreous membrane caused by a $S$. epidermidis infection.

After the removal of the intraocular lens the ocular inflammation in this case responded rapidly to systemic antibiotic therapy. With the exception of 1 case reported by Forster ${ }^{3}$ all of the previous reported cases of Staphylococcus epidermidis endophthalmitis were treated with pars plana vitrectomy, with the injection of intravitreal gentamicin and cephaloridine. There have been no clinical series reporting the results of the treatment of endophthalmitis caused by this organism with topical and systemic medication alone, though in a study of experimental Staphylococcus epidermidis endophthalmitis Cottingham and Forster ${ }^{7}$ showed this organism to be responsive to topical or intraocular antibiotics as well as to vitrectomy alone. The rapid response of our case to topical and systemic antibiotic therapy suggests that vitrectomy may not be needed in some of these cases.

This case re-emphasises the fact that with an endophthalmitis caused by an organism of low virulence the clinical signs can be masked by topical corticosteroid medication. Additionally, topical corticosteroid medication impairs the ocular immunological defence against the organism, resulting in unchecked growth of any bacterial population.

The possibility of a low-grade endophthalmitis due to Staphylococcus epidermidis or other organism of low virulence should be considered in any case of persistent postoperative inflammation, especially in the presence of corticosteroid treatment, and this clinical presentation should lead the surgeon to prompt culture of a vitreous aspirate.

This work was supported in part by a Public Health Service grant F 32 EY 05357-01 (Dr Schanzlin).

\section{References}

1 Theodore FH. Bacterial endophthalmitis after cataract extraction. Int Ophthalmol Clin 1964; 4: 839-59.

2 Valenton MJ, Brubaker RF, Allen HF. Staphylococcus epidermidis (albus) endophthalmitis. Arch Ophthalmol 1973; 89: 94-6.

3 Forster RK, Zachary IG, Cottingham AJ, Norton EWD. Further observations on the diagnosis, causes, and treatment of endophthalmitis. Am J Ophthalmol 1976; 81 : 52-6.

4 Eichenbaum DM, Jaffe NS, Clayman HM, Light DS. Pars plana vitrectomy as a primary treatment of bacterial endophthalmitis. Am J Ophthalmol 1978; 86: 167-71.

5 Allen HF, Grove AS. Early acute aseptic iritis after cataract extraction. Trans Am Acad Ophthalmol Otolaryngol 1976; 81 : 145-50.

6 Kohn AN. Bacterial cultures of lenses removed during cataract surgery. Am J Ophthalmol 1978; 86: 162-6.

7 Cottingham AJ, Forster RK. Vitrectomy in endophthalmitis. Arch Ophthalmol 1976; 94: 2078-81. 